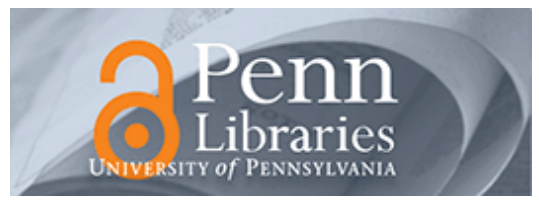

University of Pennsylvania ScholarlyCommons

October 1999

\title{
Nature of Viscoelasticity in Lamellar Block Copolymers: Contraction Correlated to Strain Localization
}

D. L. Polis

University of Pennsylvania

Karen I. Winey

University of Pennsylvania, winey@lrsm.upenn.edu

A. J. Ryan

University of Sheffield

S. D. Smith

The Procter and Gamble Company

Follow this and additional works at: https://repository.upenn.edu/mse_papers

\section{Recommended Citation}

Polis, D. L., Winey, K. I., Ryan, A. J., \& Smith, S. D. (1999). Nature of Viscoelasticity in Lamellar Block Copolymers: Contraction Correlated to Strain Localization. Retrieved from https://repository.upenn.edu/ mse_papers/88

Copyright American Physical Society. Reprinted from Physical Review Letters, Volume 83, Issue 14, October 1999, pages 2861-2864.

Publisher URL: http://dx.doi.org/10.1103/PhysRevLett.83.2861

This paper is posted at ScholarlyCommons. https://repository.upenn.edu/mse_papers/88

For more information, please contact repository@pobox.upenn.edu. 


\title{
Nature of Viscoelasticity in Lamellar Block Copolymers: Contraction Correlated to Strain Localization
}

\author{
Abstract \\ We determined the local strain profile in sheared lamellar block copolymers. A trilayer model, based on \\ elastic brushes separated by viscous interpenetration zones, captures the rheological response of these \\ materials and provides a measure of the relative contributions of elastic versus viscous strain. The elastic \\ chain distortions were evident from a reversible lamellar contraction, as measured from in situ small- \\ angle $x$-ray scattering. The contraction was directly correlated to the elastic strain from our trilayer model; \\ three distinct diblock copolymers conform to the predicted relationship, thus suggesting universal \\ behavior.
}

\section{Comments}

Copyright American Physical Society. Reprinted from Physical Review Letters, Volume 83, Issue 14, October 1999, pages 2861-2864.

Publisher URL: http://dx.doi.org/10.1103/PhysRevLett.83.2861 


\title{
Nature of Viscoelasticity in Lamellar Block Copolymers: Contraction Correlated to Strain Localization
}

\author{
D. L. Polis and K. I. Winey* \\ Laboratory for Research on the Structure of Matter, Department of Materials Science and Engineering, \\ University of Pennsylvania, Philadelphia, Pennsylvania 19104-6272 \\ A. J. Ryan \\ Department of Chemistry, University of Sheffield, Sheffield S3 7HF, United Kingdom \\ S. D. Smith \\ Corporate Research Division, The Procter and Gamble Company, Cincinnati, Ohio 45239-8707
}

(Received 20 May 1999)

\begin{abstract}
We determined the local strain profile in sheared lamellar block copolymers. A trilayer model, based on elastic brushes separated by viscous interpenetration zones, captures the rheological response of these materials and provides a measure of the relative contributions of elastic versus viscous strain. The elastic chain distortions were evident from a reversible lamellar contraction, as measured from in situ small-angle $\mathrm{x}$-ray scattering. The contraction was directly correlated to the elastic strain from our trilayer model; three distinct diblock copolymers conform to the predicted relationship, thus suggesting universal behavior.

PACS numbers: $83.70 . \mathrm{Hq}, 61.10 . \mathrm{Eq}, 83.10 . \mathrm{Nn}$
\end{abstract}

Lamellae-forming block copolymer melts are selfassembled polymer brushes, organized in such a way that opposing brushes interpenetrate in the center of each lamella [Fig. 1(a)]. In strongly segregated block copolymers, the junction points between $A$ and $B$ blocks are confined to a narrow interfacial regime and the blocks are stretched relative to their unperturbed dimensions [1]. Researchers have shown that this self-assembled nanostructure gives rise to distinctive viscoelastic properties that are common to layered, soft materials [2]. The microscopic origin of viscoelasticity in block copolymers has been addressed theoretically by focusing on either the elastic or the viscous contributions. Williams and MacKintosh propose that the opposing polymer brushes, which comprise a single composition microdomain, are distorted elastically by shear strain [3]. They predict that these elastic distortions give rise to a lamellar contraction. Witten and co-workers [4] and Joanny [5] suggest that the viscous response of lamellar block copolymers is dominated by slip in the interpenetration zone, where opposing brushes meet. Neither of these microscopic theories have been verified experimentally. Furthermore, the relative magnitude of these two effects is unknown because local strain profiles have not been determined.

In this Letter, we show that both elastic chain distortions and viscous brush slip occur in lamellar microdomains. The elastic chain distortions manifest themselves in a shear-induced lamellar contraction, as predicted by Williams and MacKintosh [3]. The contraction is measured during simultaneous rheology and small-angle $\mathrm{x}$-ray scattering (SAXS) for three different copolymers. We propose a trilayer model to describe the observed rheological response, in which elastic polymer brushes are distinguished from viscous interpenetration zones, thus combining the concepts from the aforementioned models. Fitting our trilayer model to the measured rheological responses of the copolymers provides a local strain profile. In other words, we determine the relative contributions of elastic chain distortions and viscous slip between brushes, as shown schematically in Fig. 1(b). Correlating the lamellar contraction to the elastic strain leads to a master curve for three copolymers, thus verifying the local strain profile. Understanding the deformation profile within the lamellae has proven to be crucial in elucidating the rheological response of block copolymers and will impact more generally that of opposing brushes.

It is well known that oscillatory shear can induce macroscopic alignment of lamellar microdomains in block copolymers [6]. This process was used to induce a single crystal-like "parallel" orientation in three copolymer samples. In this parallel orientation the normal to the lamellae is aligned parallel to the velocity-gradient direction [6]. A poly(styrene- $b$-methyl methacrylate) diblock copolymer and two poly(styrene- $b$-ethylene propylene) diblock copolymers were examined, which we denote SMMA(36-29), SEP(38-62), and SEP(39-24), indicating the respective nominal average molecular weights of the blocks $\left(\times 10^{3} \mathrm{~g} / \mathrm{mol}\right)$. Each of the block copolymers has a similar molecular weight styrene block, $\sim 38000 \pm$ $2000 \mathrm{~g} / \mathrm{mol}$; however, the second blocks differ in either composition and/or molecular weight. Following the prealignment, each specimen was deformed at a constant shear rate, i.e., steady-shear deformation, of $0.005 \mathrm{~s}^{-1}$ to a strain of $75 \%$ at $180{ }^{\circ} \mathrm{C}$. 

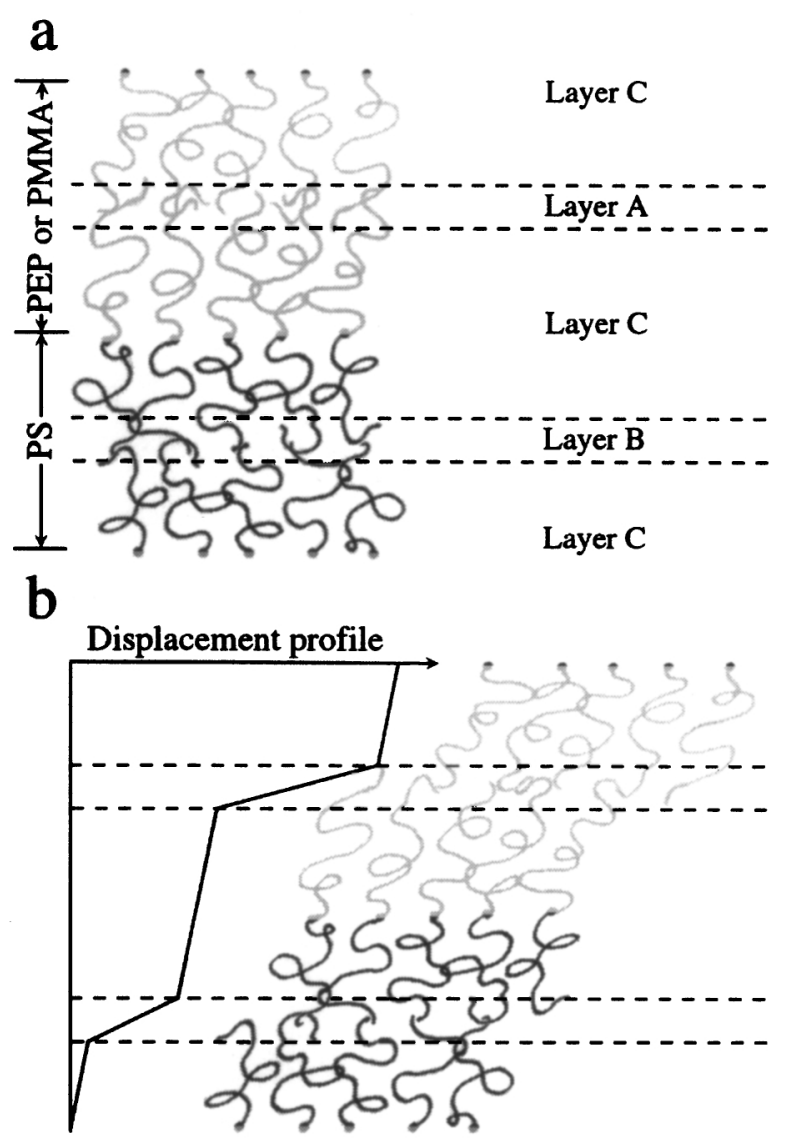

FIG. 1. (a) Schematic of a lamellar diblock copolymer. The dotted lines separate the diblock copolymers by viscoelastic properties: $(A)$ the interpenetration zone within the PEP or PMMA domains, $(B)$ the interpenetration zone within the PS domains, and $(C)$ the remaining block copolymer segments. (b) Schematic showing lamellae during steady shear and the respective deformation profile. Considerable slip occurs in the interpenetration zones (layers $A$ and $B$ ), whereas layer $C$ shows modest strain in the form of chain tilting and stretching.

SAXS and rheology were used simultaneously to measure the lamellar contraction and stress during steady shear in the three specimens. These in situ experiments are described in more detail elsewhere, in which we characterize the orientation of lamellae as a function of strain [7]. In order to obtain scattered intensity $(I)$ versus momentum transfer $\left(q=4 \pi \lambda^{-1} \sin \theta\right)$ for the parallel orientation, 2D patterns were integrated over a $30^{\circ}$ azimuthal angle $(\mu)$, centered on the parallel orientation $\left(\mu=0^{\circ}\right)$. These scattering profiles, with the background manually subtracted, clearly exhibit multiple order Bragg peaks from the lamellar morphology, showing up to the eighth order peak in the case of SEP(38-62). To obtain accurate peak positions, Gaussian peaks were fit to the multiple order Bragg peaks. From the peak positions, a plot of $D_{n}$ vs $1 / n$ was constructed, where $n$ is the order of the Bragg peak and $D_{n}$ is the corresponding spacing $\left(D_{n}=2 \pi / q_{n}\right)$. The lamellar spacing $(D)$ was determined using linear regression, specifically, $D_{n}=m(1 / n)+b$, where $m=D$ and $b=0$. Each reported lamellar spacing was determined from the average of ten scattering patterns collected over $15 \%$ strain intervals. These data were normalized by the lamellar periods prior to steady shear, giving a reduced lamellar period $D\left(e_{12}\right) / D(0)$, where $e_{12}$ is the macroscopic shear strain. $D\left(e_{12}\right) / D(0)$ vs $e_{12}$ is shown in Fig. 2, in which $D\left(e_{12}\right) / D(0)$ decreases monotonically with $e_{12}$ for each copolymer. The lamellar contraction was more dramatic for SEP(38-62) reaching 3\% after 75\% strain, as opposed to a $1.3 \%$ and $0.3 \%$ contraction in $\operatorname{SMMA}(36-29)$ and SEP(39-24), respectively. Note that the lamellar contraction is reversible, such that when sheared in the opposite direction the lamellar period returns to $D(0)$ as the strain returns to $0 \%$. Although a lamellar contraction was predicted by Williams and MacKintosh [3], their theory predicts that the reduced period should show universal behavior, which is contrary to Fig. 2.

Williams and MacKintosh proposed a model for shearing diblock copolymers with a parallel orientation of lamellae [3]. Focusing on deformation rates that are fast relative to molecular diffusion, they assume that the polymer brushes respond elastically. Furthermore, they assume the deformation profile is uniform throughout a macroscopic sample, leading to uniform chain tilting and stretching. Their theory is based on a free energy description of the system, such that chains adopt an equilibrium conformation during the deformation. This new conformation, tilted with respect to the lamellar normal, leads to an increase in the interfacial area per chain and a decrease in the lamellar period. The lamellar contraction is a result of the competition between a reduction of the chain stretching energy versus an increase in the interfacial energy. Their theory predicts that the relative lamellar contraction is solely a function of the strain in the polymer brushes. Therefore, if lamellar block copolymers deform elastically and the strain is uniform throughout the lamellae, then all

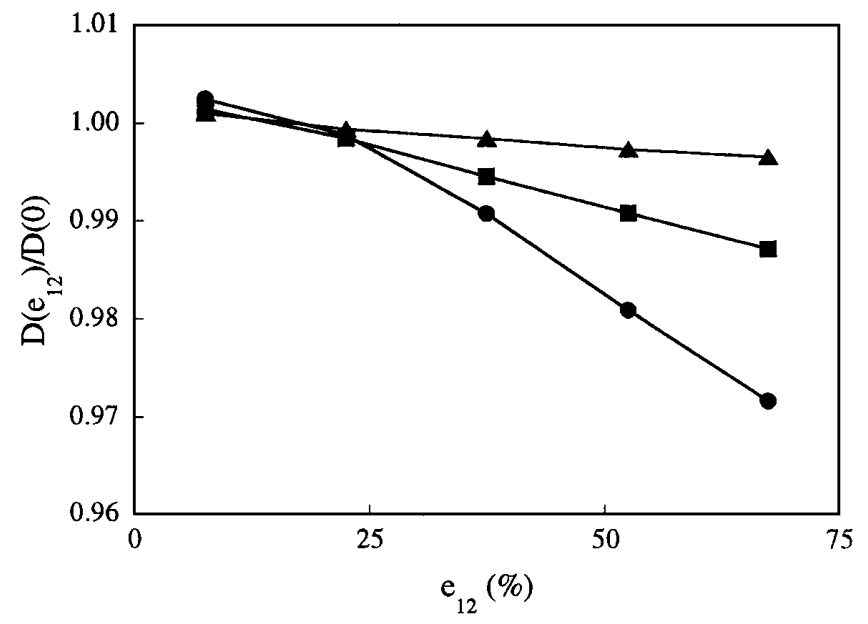

FIG. 2. Relative lamellar period, $D\left(e_{12}\right) / D(0)$, versus macroscopic strain, $e_{12}$, for $\operatorname{SEP}(38-62)$, SEP(39-24), and SMMA(3629 ) indicated by circles, triangles, and squares, respectively. 
three materials should exhibit the same relative contraction as a function of the macroscopic strain.

In contrast, Witten, Leibler, and Pincus [4] suggest that the central region of a microdomain, where like-brushes meet in the interpenetration zone, is where most of the viscous dissipation occurs in lamellar diblock copolymers. Consequently, the deformation profile may not be uniform throughout a single microdomain, contrary to the deformation profile assumed by Williams and MacKintosh [3]. Joanny extended this idea by theoretically examining the viscous friction between two polymer brushes rubbed against each other [5]. He found that the small region where the opposing brushes interpenetrate dominated the viscous dissipation. Furthermore, he determined that shearing interpenetrating brushes leads to chain tilting and stretching, such that the interpenetration zone between opposing brushes becomes smaller. In other words, shearing reduces the degree of entanglement in the interpenetration zone.

In order to reconcile our contraction data (Fig. 2) with the predictions of Williams and MacKintosh [3], we consider both the elastic and viscous nature of block copolymers and propose a trilayer model. Rather than dividing the lamellar diblock copolymer into two layers based on composition, we divide the system into three layers based on viscoelastic characteristics, Fig. 1(a). As proposed by Witten and co-workers [4] and Joanny [5], we assume that the interpenetration zones, layers $A$ and $B$, are responsible for the viscous dissipation in these lamellar diblock copolymers. In contrast, we assume the deformation in layer $C$ is largely due to elastic-type chain distortions, leading to the observed lamellar contraction as predicted by Williams and MacKintosh [3]. Based on these assumptions, a simple constitutive relationship for an elastic layer (layer $C$ ) lubricated by two viscous layers (layers $A$ and $B$ ) is constructed. The mechanical responses of layers $A$ and $B$ are described by Newton's law for linear viscous fluids, while Hooke's law for linear elastic solids is used to describe layer $C$.

Lamellae in the parallel orientation are such that the shear stress in each layer $i$ is equal to the macroscopic shear stress, $\sigma_{12, i}=\sigma_{12}$. The macroscopic strain rate, which is a constant in our experiments $(\dot{\gamma})$, is equal to the sum of the microscopic strain rates in each layer weighted by the respective volume fractions of the layers, $\phi_{i}$, such that

$$
\dot{\gamma}=\frac{d e_{12}}{d t}=\sum_{i=A, B, C} \phi_{i} \frac{d e_{12, i}}{d t} .
$$

One can solve for the stress-strain relationship by substituting the constitutive relations for the three layers into Eq. (1). Applying the boundary condition that stress is identically zero at $0 \%$ strain gives

$$
\sigma_{12}=\dot{\gamma} \eta_{\mathrm{eff}}\left[1-\exp \left\{-\frac{G_{C} / \phi_{C}}{\eta_{\mathrm{eff}}}\left(\frac{e_{12}}{\dot{\gamma}}\right)\right\}\right],
$$

where

$$
\eta_{\mathrm{eff}}=\frac{\eta_{A} \eta_{B}}{\phi_{B} \eta_{A}+\phi_{A} \eta_{B}} .
$$

Thus, shearing a parallel array of elastic polymer brushes separated by viscous interpenetration zones should result in a Maxwell-type stress-strain response as given by Eq. (2). In this model the stress asymptotically approaches a limit $\dot{\gamma} \eta_{\text {eff }}$ dictated by the effective viscosity. The rate of approach is controlled by the ratio of the effective modulus of layer $C$, which is composed of two compositionally different layers, to the effective viscosity, $G_{C} / \eta_{\text {eff }}$.

The stress-strain response for the three copolymers is shown in Fig. 3, along with the best fit of Eq. (2). Equation (2) reasonably captures the rheological response for the three copolymers, especially considering that the samples are not true single crystals of parallel lamellae [8]. Fitting the rheological data gives $\eta_{\text {eff }}$ and $G_{C} / \phi_{C}$. $G_{C}$ can be extracted by estimating the relative sizes of the three layers using a model for the static configuration of the block copolymer chain to determine $\phi_{C}$ [9]: $\operatorname{SMMA}(36-29) \quad \phi_{C}=0.66 ; \quad \operatorname{SEP}(38-62) \quad \phi_{C}=0.72$; $\operatorname{SEP}(39-24) \phi_{C}=0.70 . \quad G_{C}$ can then be used to determine the strain in the polymer brushes $\left(e_{12, C}=\sigma_{12} / G_{C}\right)$ for a particular macroscopic strain $e_{12}$. Therefore, the lamellar contraction data can be reevaluated in terms of a brush contraction versus the strain in the polymer brush, $D_{C}\left(e_{12, C}\right) / D_{C}(0)$, where $D_{C}(0)=\phi_{C} D(0)$. Figure 4 shows $D_{C}\left(e_{12, C}\right) / D_{C}(0)$ vs $e_{12, C}$ for the three copolymers. The relative contractions of the three copolymers fall on a master curve when examined in terms of the local strain in the brushes, qualitatively consistent with the prediction of Williams and MacKintosh [3]. This supports

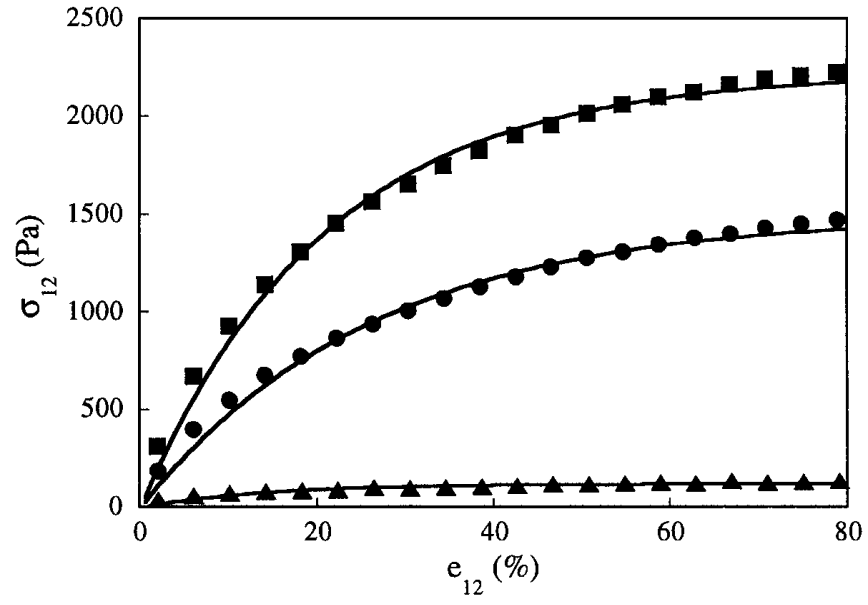

FIG. 3. Stress $\left(\sigma_{12}\right)$ versus strain $\left(e_{12}\right)$ for $\operatorname{SEP}(38-62)$, SEP(39-24), and SMMA(36-29) indicated by circles, triangles, and squares, respectively. The solid lines represent the best fit of Eq. (2) to the rheological data. The calculated values of $\eta_{\text {eff }}$ and $G_{C}$ are in good agreement with values available from dynamic mechanical testing. Furthermore, the relative values for the three materials follow reasonable trends, as will be presented at length in a forthcoming paper. 


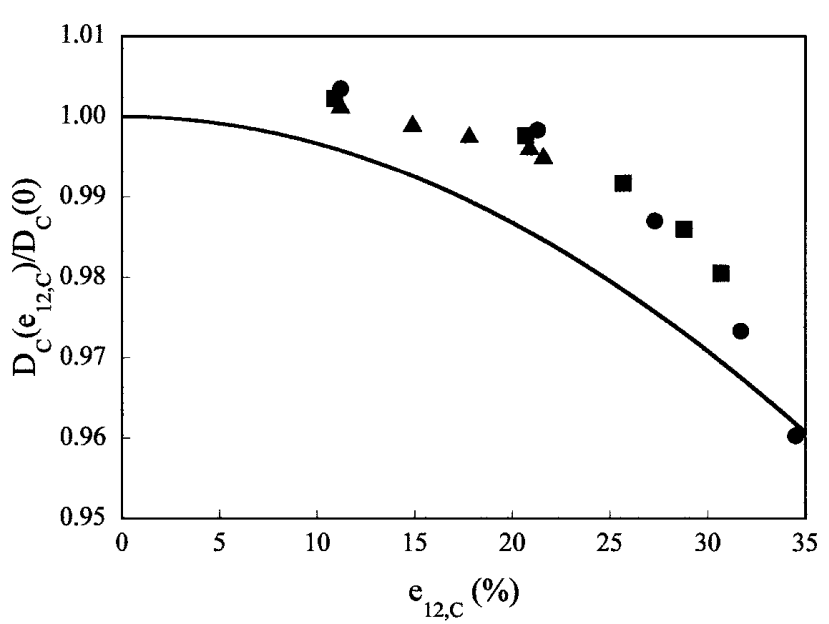

FIG. 4. Master curve, relating the relative brush height, $D_{C}\left(e_{12, C}\right) / D_{C}(0)$, and the localized strain, $\left(e_{12, C}\right)$, in layer $C$ for SEP(38-62), SEP(39-24), and SMMA(36-29) indicated by circles, triangles, and squares, respectively. The solid line shows the master curve prediction of Williams and MacKintosh [3]. While no values of $\phi_{C}$ produce quantitative agreement with the Williams and MacKintosh model, the $\phi_{C}$ values determined from the Rubinstein and Obukhov model [9] provide the best qualitative agreement.

their assumption that the chains adopt an equilibrium conformation during a shear deformation, which in this case is expected to be fast relative to polymer diffusion parallel to the lamellae [10]. More importantly, the master curve strongly supports the idea that a considerable portion of the macroscopic deformation is localized in the interpenetration zones, where brush-on-brush slip occurs, as illustrated in Fig. 1(b). A preferred slip plane is found in all systems that exhibit kink band defects, including hexagonally packed crystalline metals, laminated rocks, layered composites, etc. Thus, the existence of a preferential slip plane parallel to the lamellae partially explains the occurrence of kink bands in block copolymers $[8,11]$ and could also contribute to shear-induced lamellar rotation [7].

We have confirmed that elastic chain distortions and viscous slip between opposing brushes are dominant microscopic effects that strongly influence the macroscopic rheological response of lamellar block copolymers. Our trilayer model, which combines these two contributions, accurately describes the stress-strain data from our steadyshear experiments. This model provides the relative magnitude of elastic versus viscous strain in the microdomains, such that strain in the polymer brushes could be calculated. The elastic strain in the brushes is substantially less than that applied macroscopically. Examining the lamellar contraction in terms of the local strain in the polymer brushes collapses the data from three copolymers onto a master curve, consistent with the predictions of Williams and MacKintosh [3]. Furthermore, the master curve supports the idea that the viscous dissipation occurs primarily in the interpenetration zone between opposing polymer brushes.

Our results on the local strain profile in lamellar microdomains have more general implications regarding polymer brushes. Because polymer brushes are especially important for surface modification, there has been considerable attention focused on shearing opposing polymer brushes [12]. However, experiments have been unable to validate some of the theoretical predictions including the perturbations of brush height during shearing. The shearinduced contraction and brush-on-brush slip presented in this Letter are consistent with recent computer simulations of interpenetrating brushes grafted to solid surfaces $[12,13]$ suggesting that the behavior may be universal.

We thank Professor D. C. Morse, University of Minnesota, for helpful discussions. Work at the University of Pennsylvania was supported by NSF-DMR-MRSEC (9632598), NSF-DMR-YIA (9457997), and Procter and Gamble. Beam time at Daresbury Laboratory, Warrington, United Kingdom, was allocated under EPSRC Grant (No. GR/M22621).

*To whom correspondence should be addressed.

[1] F. S. Bates and G. H. Fredrickson, Annu. Rev. Phys. Chem. 41, 525-557 (1990).

[2] R. G. Larson, K. I. Winey, S. S. Patel, H. Watanabe, and R. Bruinsma, Rheol. Acta 32, 245-253 (1993).

[3] D. R. M. Williams and F. C. MacKintosh, Macromolecules 27, 7677-7680 (1994).

[4] T.A. Witten, L. Leibler, and P.A. Pincus, Macromolecules 23, 824-829 (1990).

[5] J. F. Joanny, Langmuir 8, 989-995 (1992).

[6] Z.R. Chen and J.A. Kornfield, Polymer 39, 4679-4699 (1998).

[7] D. L. Polis, S.D. Smith, N.J. Terrill, A. J. Ryan, D. C. Morse, and K. I. Winey, Macromolecules 32, 4668-4676 (1999).

[8] D. L. Polis and K. I. Winey, Macromolecules 31, 36173625 (1998).

[9] M. Rubinstein and S.P. Obukhov, Macromolecules 26, 1740-1750 (1993).

[10] T.P. Lodge and M.C. Dalvi, Phys. Rev. Lett. 75, 657660 (1995).

[11] D. L. Polis and K. I. Winey, Macromolecules 29, 81808187 (1996).

[12] G. S. Grest, in Polymers in Confined Environments, edited by S. Granick (Springer-Verlag, Berlin, 1999), Vol. 138, pp. $150-180$.

[13] I. M. Neelov, O. V. Borisov, and K. Binder, J. Chem. Phys. 108, 6973-6988 (1998). 